\title{
Conversion therapy of gastric cancer with massive malignant ascites and ovarian metastases by systemic and intraperitoneal chemotherapy
}

\author{
TOMOHIRO KONDO $^{1}$, HIROMITSU KITAYAMA ${ }^{1}$, JUNKO SUGIYAMA ${ }^{1}$, \\ MICHIAKI HIRAYAMA ${ }^{2}$, YOSHINORI SUZUKI ${ }^{3}$, YUMIKO OYAMADA ${ }^{4}$ and YASUSHI TSUJI ${ }^{1}$ \\ Departments of ${ }^{1}$ Medical Oncology, ${ }^{2}$ Gastroenterology, ${ }^{3}$ Surgery and ${ }^{4}$ Surgical Pathology, \\ Tonan Hospital, Sapporo, Hokkaido 060-0001, Japan
}

Received August 13, 2016; Accepted September 23, 2016

DOI: $10.3892 / \mathrm{mco} .2016 .1058$

\begin{abstract}
Intravenous and intraperitoneal paclitaxel with S-1 is showing promising results in gastric cancer with peritoneal metastases. We herein report a successful conversion of unresectable to resectable disease using combination chemotherapy with trastuzumab. The patient was a 39 -year-old woman with human epidermal growth factor receptor 2-positive gastric cancer with peritoneal, pulmonary and bilateral ovarian metastases. After 6 cycles of S-1 plus cisplatin with trastuzumab, followed by 15 cycles of intravenous and intraperitoneal paclitaxel with S-1 and trastuzumab, the pulmonary and peritoneal metastases exhibited complete response and no evidence of malignancy was found on diagnostic laparoscopy. We performed metastasectomy of the bilateral sizeable ovaries, followed by total gastrectomy. The patient had no recurrence for 16 months after the gastrectomy. Therefore, satisfactory response to systemic and intraperitoneal chemotherapy may convert unresectable to resectable disease, and primary tumor resection with ovarian metastasectomy may prolong survival. This combination chemotherapy has the potential of becoming a conversion therapy for gastric cancer with peritoneal metastases, even if ascites and ovarian metastases are extensive.
\end{abstract}

\section{Introduction}

Gastric cancer with peritoneal metastases has a poor prognosis (1). S-1 plus cisplatin is widely administered for metastatic or unresectable gastric cancer and is considered to be the standard first-line regimen in Japan (2). Recently, intravenous (IV) and intraperitoneal (IP) administration of paclitaxel (PTX)

Correspondence to: Dr Tomohiro Kondo, Department of Medical Oncology, Tonan Hospital, Kita 1 Jo Nishi 6 Chome, Chuo-ku, Sapporo, Hokkaido 060-0001, Japan

E-mail:kondot@kuhp.kyoto-u.ac.jp

Key words: gastric cancer, peritoneal metastases, intraperitoneal chemotherapy, conversion therapy, paclitaxel with S-1 is showing promising results for gastric cancer with peritoneal metastases (3). However, it remains unclear whether conversion of unresectable gastric cancer to resectable disease is possible with this combination chemotherapy. We herein present a case of a successful conversion therapy using a combination of systemic and IP chemotherapy, followed by ovarian metastasectomy and primary tumor resection.

\section{Case report}

A 39-year old woman presented with abdominal distention and pain. The patient was diagnosed with advanced gastric cancer with pulmonary metastases, massive malignant ascites and bilateral ovarian metastases (Fig. 1A). Gastrointestinal endoscopy revealed a Borrmann type IV tumor and the biopsy revealed poorly differentiated adenocarcinoma overexpressing the human epidermal growth factor receptor 2 (HER-2) protein (3+) (Fig. 2). Triweekly SPT therapy was administered, which consisted of oral S-1, IV cisplatin and IV trastuzumab (4). After 2 cycles of the therapy, computed tomography (CT) and gastrointestinal endoscopy revealed disappearance of the malignant ascites and complete response of the pulmonary metastases (Fig. 1B). After an additional 4 cycles of the SPT therapy, diagnostic laparoscopy was conducted. As residual peritoneal metastases were found, a peritoneal access device was implanted in the right lower abdomen wall and a catheter was placed in the pelvic cavity (Fig. 3A). Therapy was then switched to IV and IP PTX with S-1 and trastuzumab. The regimen was as follows: $\mathrm{S}-180 \mathrm{mg} / \mathrm{m}^{2} /$ day for 14 consecutive days followed by 7 days of rest, IV PTX at $50 \mathrm{mg} / \mathrm{m}^{2}$ and IP PTX at $20 \mathrm{mg} / \mathrm{m}^{2}$ on days 1 and 8 , and triweekly IV trastuzumab at the standard dose on day 1. Gastrointestinal endoscopy and CT after 11 cycles of this combination chemotherapy confirmed no progression of the primary lesion and metastatic disease (Fig. 1C). Secondary laparoscopy revealed multiple white nodules without evidence of malignancy and peritoneal lavage cytology was negative. Bilateral ovarian metastasectomy was conducted in order to prevent ovarian torsion due to the extensive metastases. The pathological examination revealed residual ovarian metastatic disease with overexpression of the HER-2 protein (3+) (Fig. 4). Following 
A

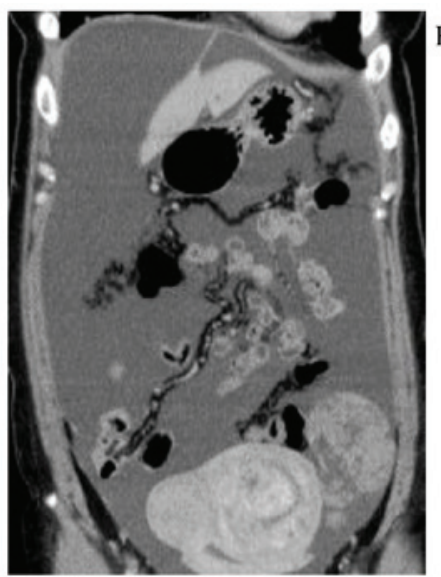

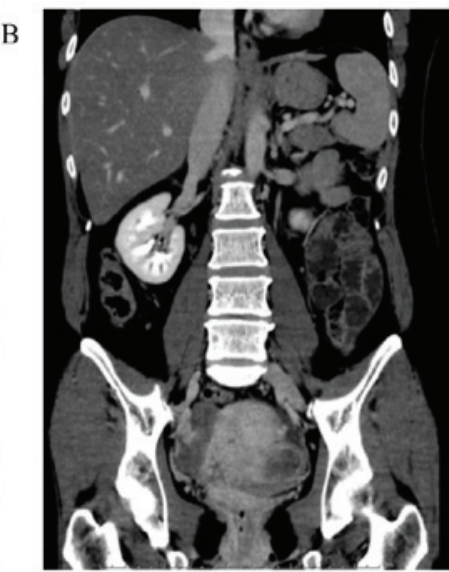

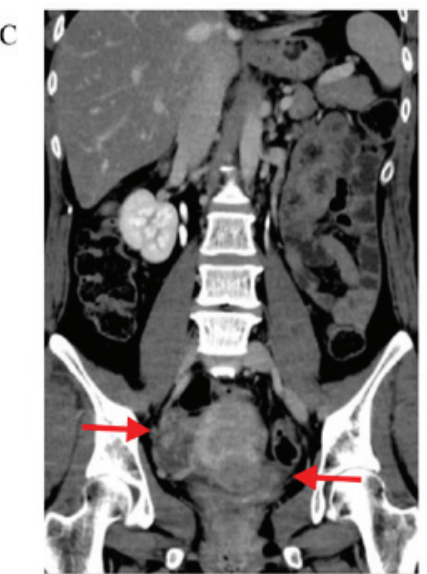

Figure 1. Abdominal computed tomography showing the efficacy of chemotherapy. (A) Massive malignant ascites and bilateral ovarian metastases were present prior to chemotherapy. (B) After 5 months of S-1, cisplatin and trastuzumab therapy, the malignant ascites disappeared. (C) After 8 months of therapy with intravenous and intraperitoneal paclitaxel with S-1 and trastuzumab, the malignant ascites had disappeared, whereas the bilateral extensive ovarian metastases persisted (arrows).

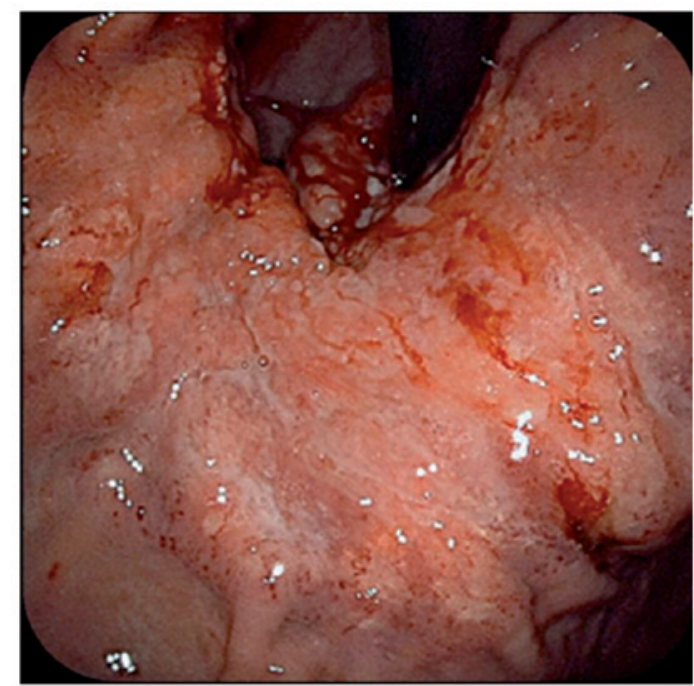

Figure 2. Gastrointestinal endoscopic findings prior to chemotherapy. A Borrmann type IV tumor occupied a large part of the lesser curvature of the gastric body. The tumor reached the gastric cardia.

ovariectomy, 4 additional cycles of the systemic and IP chemotherapy were administered. As no obvious non-curative factors were identified, laparoscopic total gastrectomy was performed after pathologically re-confirming disappearance of the peritoneal metastases and negative peritoneal lavage cytology (Fig. 3B). The postoperative course was uneventful.

The final diagnosis revealed minute residual tumor cell nests at the primary site (stomach) with overexpression of HER-2 (3+) (Fig. 5). The pathological diagnosis was as follows: U, Less, ypType4, por2, ypT2, sci, INFc, ly0, v0, ypN0 (0/41), pPM0 (12 mm), pDM0 (147 mm), histological grade 2 according to the Third Edition of Japanese Classification of Gastric Carcinoma (5). The patient continued the combination chemotherapy with S-1 and IP PTX (changed from the original IV and IP paclitaxel) with trastuzumab for 1 year after the gastrectomy, suffering mild adverse effects, such as low-grade anorexia. Chemotherapy was then discontinued according to the patient's wishes, and the patient developed no recurrence

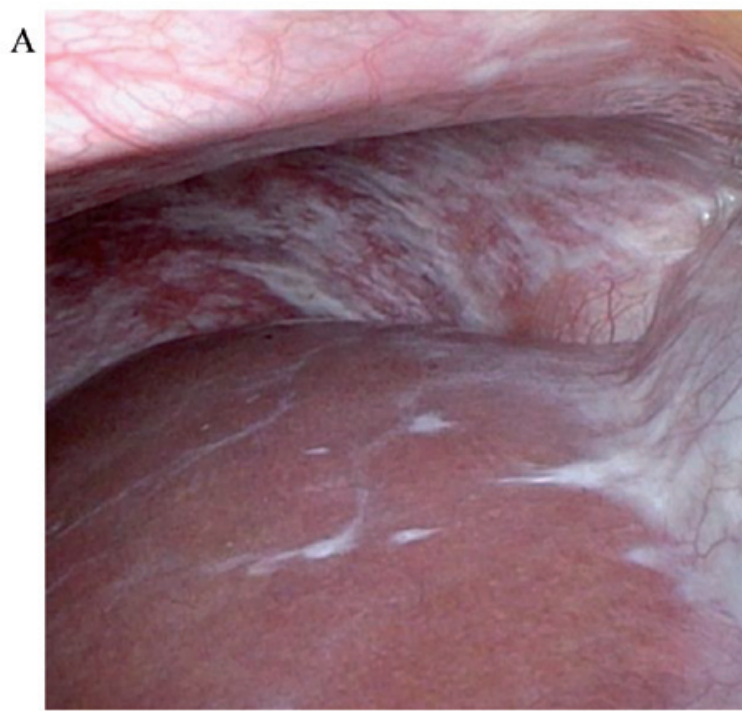

B

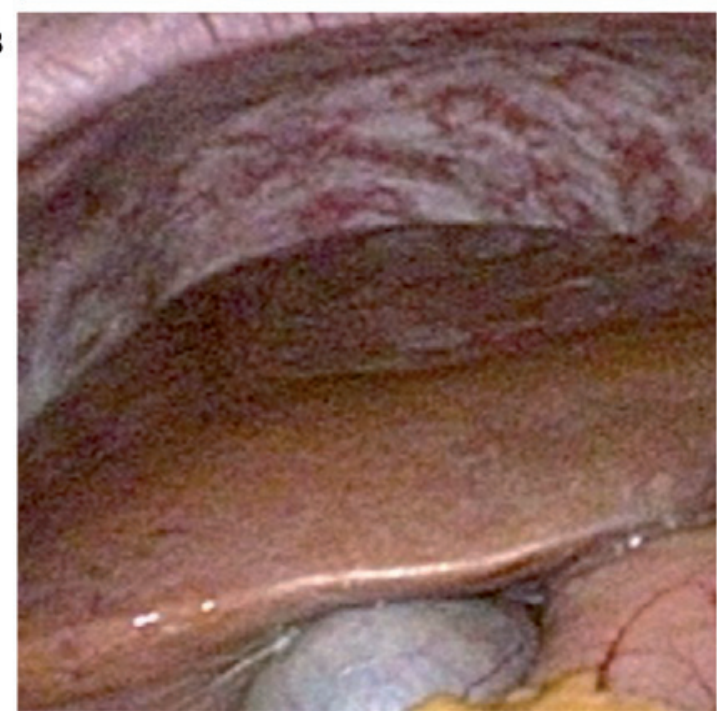

Figure 3. Findings of diagnostic laparoscopy after each chemotherapy cycle. (A) After 5 months of S-1 plus cisplatin with trastuzumab, several residual white nodules in the peritoneum were pathologically diagnosed as peritoneal metastases. (B) After switching to intravenous and intraperitoneal paclitaxel with S-1 and trastuzumab, these nodules became fibrotic, without evidence of malignancy. 


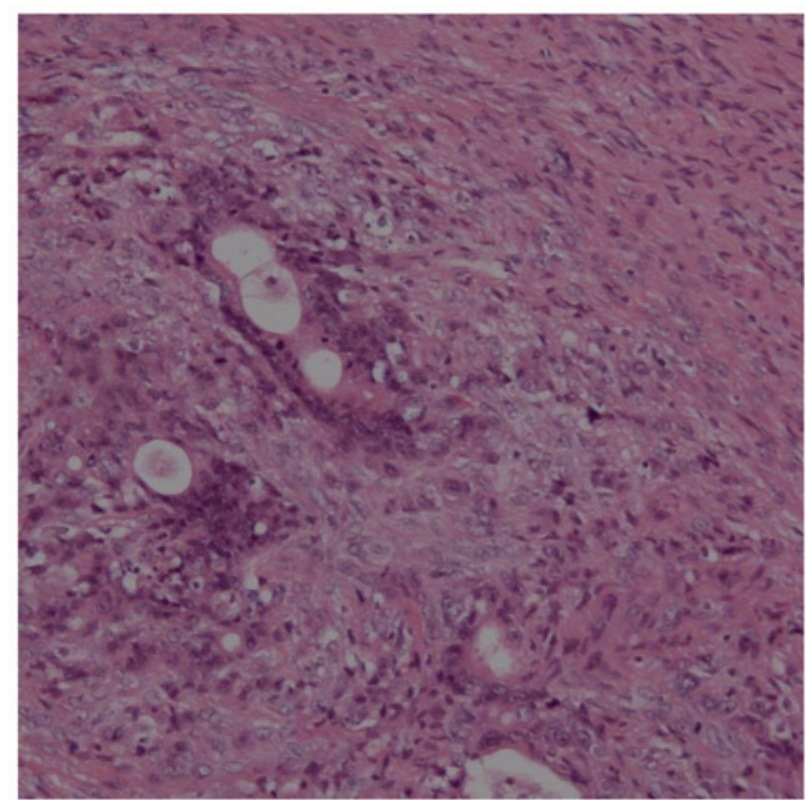

Figure 4. Microscopic findings of ovarian metastases. Extensive metastases from gastric cancer persisted after 5 months of S-1 plus cisplatin with trastuzumab, followed by 8 months of intravenous and intraperitoneal paclitaxel with S-1 and trastuzumab. Hematoxylin and eosin staining; magnification, x100.

A

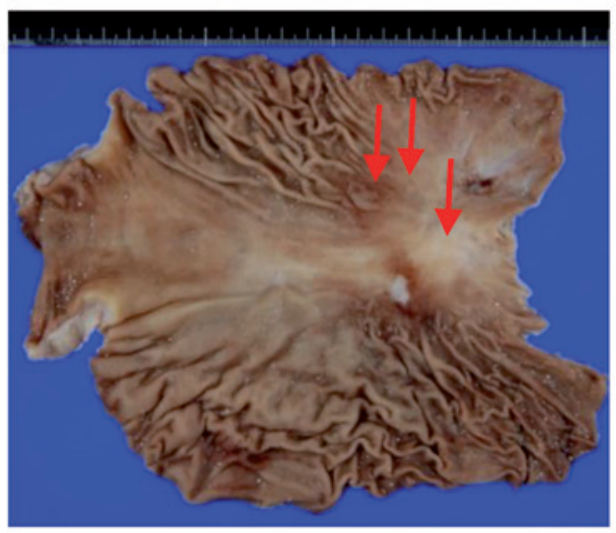

B

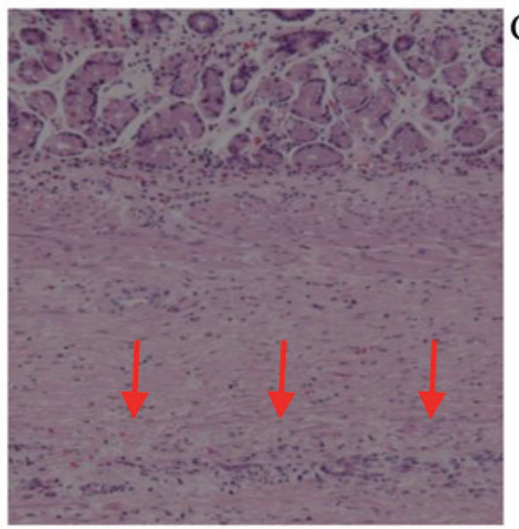

$\mathrm{C}$

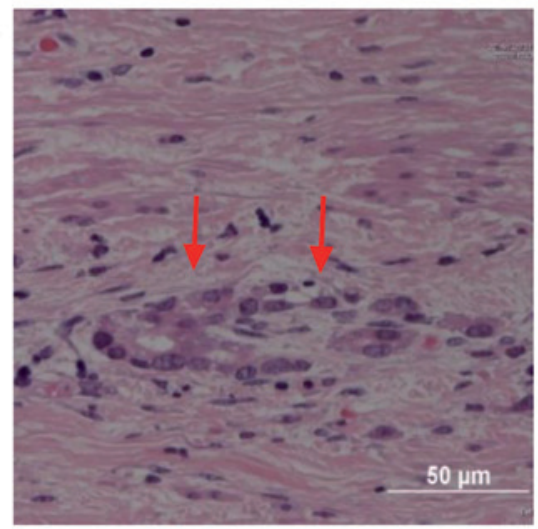

Figure 5. (A) Macroscopic and (B and C) microscopic findings of the stomach after chemotherapy. Only a few residual cancer cell nests remained in the submucosal layer (arrows). (B and C) Hematoxylin and eosin staining; magnification: B x100 and C, x400.

for 16 months from the gastrectomy and 33 months from diagnosis. However, 4 months after discontinuing chemotherapy, isolated para-aortic lymph node recurrence was detected.

\section{Discussion}

Based on this case, two important clinical issues must be discussed: First, a satisfactory response to systemic and IP chemotherapy may convert unresectable gastric cancer with peritoneal metastases to resectable disease. Primary tumor resection and ovarian metastasectomy may prolong patient survival if the combination chemotherapy is sufficiently effective and there are no unresectable metastases.

The disease responded well to systemic and IP chemotherapy and was converted from unresectable to resectable. The ability of IP PTX to maintain a high concentration for prolonged periods of time contributed to the high antitumor effect against peritoneal metastases, and the tumor responded well to the drug combination of IV and IP PTX with S-1 and trastuzumab. A phase II trial demonstrated that IV and IP PTX with S-1 were well-tolerated and highly effective in gastric cancer patients with peritoneal metastases (3). The 1-year overall survival rate was $77 \%$, and malignant ascites disappeared or was reduced in 15 of $22(68 \%)$ patients. PTX is an effective agent for gastric cancer, with a relatively good response of diffuse-type adenocarcinoma, which accounts for the majority of peritoneal metastases of this disease (6). IP administration of PTX was developed to reinforce the drug effect on peritoneal metastases (7). This is accomplished by allowing PTX to act directly on nodules at a high concentration. When PTX is administered IP, it is absorbed slowly due to its high molecular weight and lipophilic properties. IP PTX is also considered to have a mild toxicity profile $(7,8)$. IP administration of PTX has been used together with systemic chemotherapy for peritoneal metastases of ovarian cancer, where it has demonstrated significant survival benefits (9). Additionally, S-1 is an oral 5-FU agent that has been 
shown to be non-inferior to infusional 5-FU in terms of overall survival in Japanese patients with metastatic gastric cancer; furthermore, trastuzumab in combination with standard cytotoxic chemotherapy for this disease improved overall survival for patients with HER2-positive disease $(10,11)$. The tumor responded well to the combination of IV and IP PTX with S-1 and trastuzumab. This combination chemotherapy was able to convert unresectable to resectable disease; however, its viability has not been sufficiently confirmed. Conversion therapy was originally well-established in colon cancer management (12); in gastric cancer, it was recently considered beneficial in some cases with effective chemotherapy and negative peritoneal washing cytology (13). If peritoneal metastases are controlled well over a long time period, primary tumor resection appears to prolong survival. In fact, almost all peritoneal metastases in this case visibly disappeared with this combination chemotherapy.

The second clinical issue is that primary tumor resection and ovarian metastasectomy may prolong survival in gastric cancer patients with peritoneal metastases if systemic and IP chemotherapy is sufficiently effective without unresectable metastases. Kitayama et al suggested criteria for primary tumor resection with IV and IP PTX with S-1 in gastric cancer (14). The criteria are as follows: i) No distant metastases except in the peritoneal area, including the ovary; ii) negative peritoneal cytology; and iii) metastatic nodules in the peritoneal cavity visibly reduced or controlled. Of the 64 patients with severe peritoneal metastases and ascites, gastrectomy was performed in 34 patients. The median survival time of the gastrectomized patients was 26.4 months, whereas that of the 30 patients who did not undergo gastrectomy was 12.1 months. Other retrospective studies demonstrated that gastrectomy and metastasectomy, including ovariectomy, may improve survival $(15,16)$. Peng et al reported that gastrectomy and absence of ascites were the two independent prognostic factors associated with longer survival in gastric cancer (16). In addition, for colorectal cancer with peritoneal metastases, the presence of ovarian metastases did not affect prognosis in patients undergoing complete cytoreductive surgery with IP chemotherapy (17). Removing microscopic residual tumor is the expected effect of the surgery. In a mouse model, IP PTX penetrates only 100-200 $\mu \mathrm{m}$ under the surface of the disseminated tumor, and its effect against massive metastases is limited $(18,19)$. Moreover, although IV and IP PTX with S-1 was highly effective, grade 2 and 3 histological response was only obtained in $7(21 \%)$ and $1(3 \%)$ patients, respectively (14). Surgical resection of extensive residual disease is required to achieve a disease-free status, as pathological complete response in gastric cancer is rare, even with highly effective chemotherapy. If systemic and IP chemotherapy effectively control peritoneal metastases over a long period of time, primary tumor resection and ovarian metastasectomy appear to prolong survival.

In summary, satisfactory response to IV and IP PTX with S-1 and trastuzumab was able to convert unresectable gastric cancer with peritoneal metastases to resectable disease, and primary tumor resection with ovarian metastasectomy may also prolong survival in such patients. This combination chemotherapy has the potential to become a conversion therapy for gastric cancer with peritoneal metastases, even if ascites and ovarian metastases are extensive. As the optimal surgical procedure has not yet been determined in such patients, further investigation is required to determine the optimal treatment strategy for gastric cancer with peritoneal metastases.

\section{Acknowledgements}

The authors would like to thank the patient for her kind cooperation, and David Hochman for reviewing the language of our article.

\section{References}

1. Sadeghi B, Arvieux C, Glehen O, Beaujard AC, Rivoire M, Baulieux J, Fontaumard E, Brachet A, Caillot JL, Faure JL, et al: Peritoneal carcinomatosis from non-gynecologic malignancies: Results of the EVOCAPE 1 multicentric prospective study. Cancer 88: 358-363, 2000.

2. Koizumi W, Narahara H, Hara T, Takagane A, Akiya T, Takagi M, Miyashita K, Nishizaki T, Kobayashi O, Takiyama W, et al: S-1 plus cisplatin versus $\mathrm{S}-1$ alone for first-line treatment of advanced gastric cancer (SPIRITS trial): A phase III trial. Lancet Oncol 9: 215-221, 2008.

3. Yamaguchi H, Kitayama J, Ishigami H, Emoto S, Yamashita H and Watanabe T: A phase 2 trial of intravenous and intraperitoneal paclitaxel combined with S-1 for treatment of gastric cancer with macroscopic peritoneal metastasis. Cancer 119: 3354-3358, 2013

4. Kurokawa Y, Sugimoto N, Miwa H, Tsuda M, Nishina S, Okuda H, Imamura H, Gamoh M, Sakai D, Shimokawa T, et al: Phase II study of trastuzumab in combination with S-1 plus cisplatin in HER2-positive gastric cancer (HERBIS-1). Br J Cancer 110: 1163-1168, 2014.

5. Japanese Gastric Cancer Association: Japanese classification of gastric carcinoma: 3rd English edition. Gastric Cancer 14: 101-112, 2011.

6. Yamada Y, Shirao K, Ohtsu A, Boku N, Hyodo I, Saitoh H, Miyata Y and Taguchi T: Phase II trial of paclitaxel by three-hour infusion for advanced gastric cancer with short premedication for prophylaxis against paclitaxel-associated hypersensitivity reactions. Ann Oncol 12: 1133-1137, 2001.

7. Markman M, Rowinsky E, Hakes T, Reichman B, Jones W, Lewis JL Jr, Rubin S, Curtin J, Barakat R, Phillips M, et al: Phase I trial of intraperitoneal taxol: A Gynecoloic Oncology Group study. J Clin Oncol 10: 1485-1491, 1992.

8. Gelderblom H, Verweij J, van Zomeren DM, Buijs D, Ouwens L, Nooter K, Stoter G and Sparreboom A: Influence of Cremophor El on the bioavailability of intraperitoneal paclitaxel. Clin Cancer Res 8: 1237-1241, 2002.

9. Armstrong DK, Bundy B, Wenzel L, Huang HQ, Baergen R, Lele S, Copeland LJ, Walker JL and Burger RA; Gynecologic Oncology Group: Intraperitoneal cisplatin and paclitaxel in ovarian cancer. N Engl J Med 354: 34-43, 2006.

10. Boku N, Yamamoto S, Fukuda H, Shirao K, Doi T, Sawaki A, Koizumi W, Saito H, Yamaguchi K, Takiuchi H, et al; Gastrointestinal Oncology Study Group of the Japan Clinical Oncology Group: Fluorouracil versus combination of irinotecan plus cisplatin versus S-1 in metastatic gastric cancer: A randomised phase 3 study. Lancet Oncol 10: 1063-1069, 2009.

11. Bang YJ, Van Cutsem E, Feyereislova A, Chung HC, Shen L, Sawaki A, Lordick F, Ohtsu A, Omuro Y, Satoh T, et al; ToGA Trial Investigators: Trastuzumab in combination with chemotherapy versus chemotherapy alone for treatment of HER2-positive advanced gastric or gastro-oesophageal junction cancer (ToGA): A phase 3, open-label, randomised controlled trial. Lancet 376: 687-697, 2010.

12. Power DG and Kemeny NE: Chemotherapy for the conversion of unresectable colorectal cancer liver metastases to resection. Crit Rev Oncol Hematol 79: 251-264, 2011.

13. Yoshida K, Yamaguchi K, Okumura N, Tanahashi $\mathrm{T}$ and Kodera Y: Is conversion therapy possible in stage IV gastric cancer: The proposal of new biological categories of classification. Gastric Cancer 19: 329-338, 2016.

14. Kitayama J, Ishigami H, Yamaguchi H, Yamashita H, Emoto S, Kaisaki S and Watanabe T: Salvage gastrectomy after intravenous and intraperitoneal paclitaxel (PTX) administration with oral S-1 for peritoneal dissemination of advanced gastric cancer with malignant ascites. Ann Surg Oncol 21: 539-546, 2014. 
15. Kim KH,Lee KW, Baek SK, Chang HJ, Kim YJ, Park DJ, Kim JH, Kim HH and Lee JS: Survival benefit of gastrectomy \pm metastasectomy in patients with metastatic gastric cancer receiving chemotherapy. Gastric Cancer 14: 130-138, 2011.

16. Peng W, Hua RX, Jiang R, Ren C, Jia YN, Li J and Guo WJ: Surgical treatment for patients with Krukenberg tumor of stomach origin: Clinical outcome and prognostic factors analysis. PLoS One 8: e68227, 2013.

17. Eveno C, Goéré D, Dartigues P, Honoré C, Dumont F, Tzanis D, Benhaim L, Malka D and Elias D: Ovarian metastasis is associated with retroperitoneal lymph node relapses in women treated for colorectal peritoneal carcinomatosis. Ann Surg Oncol 20: 491-496, 2013
18. Soma D, Kitayama J, Konno T, Ishihara K, Yamada J, Kamei T, Ishigami H, Kaisaki S and Nagawa H: Intraperitoneal administration of paclitaxel solubilized with poly(2-methacryloxyethyl phosphorylcholine-co n-butyl methacrylate) for peritoneal dissemination of gastric cancer. Cancer Sci 100: 1979-1985, 2009.

19. Kamei T, Kitayama J, Yamaguchi H, Soma D, Emoto S, Konno T, Ishihara K, Ishigami H, Kaisaki S and Nagawa H: Spatial distribution of intraperitoneally administrated paclitaxel nanoparticles solubilized with poly (2-methacryloxyethyl phosphorylcholineco n-butyl methacrylate) in peritoneal metastatic nodules. Cancer Sci 102: 200-205, 2011. 